\title{
Efficient representation of texture details in medical images by fusion of Ripplet and DDCT transformed images
}

\author{
Ayush Dogra*, Sunil Agrawal and Bhawna Goyal \\ Department of Electronics and Communications, Panjab University, Chandigarh, India \\ *For correspondence: Email: ayush123456789@gmail.com
}

Received: 20 March 2016

Revised accepted: 1 June 2016

\begin{abstract}
Purpose: To evaluate and compare the performance of Ripplet Type-1 transform and directional discrete cosine transform (DDCT) and their combinations for improved representation of MRI images while preserving its fine features such as edges along the smooth curves and textures.

Methods: In a novel image representation method based on fusion of Ripplet type-1 and conventional/directional DCT transforms, source images were enhanced in terms of visual quality using Ripplet and DDCT and their various combinations. The enhancement achieved was quantified on the basis of peak signal to noise ratio (PSNR), mean square error (MSE), structural content (SC), average difference $(A D)$, maximum difference $(M D)$, normalized cross correlation (NCC), and normalized absolute error (NAE). To determine the attributes of both transforms, these transforms were combined to represent the entire image as well. All the possible combinations were tested to present a complete study of combinations of the transforms and the contrasts were evaluated amongst all the combinations. Results: While using the direct combining method (DDCT) first and then the Ripplet method, a PSNR value of 32.3512 was obtained which is comparatively higher than the PSNR values of the other combinations. This novel designed technique gives PSNR value approximately equal to the PSNR's of parent techniques. Along with this, it was able to preserve edge information, texture information and various other directional image features. The fusion of DDCT followed by the Ripplet reproduced the best images.

Conclusion: The transformation of images using Ripplet followed by DDCT ensures a more efficient method for the representation of images with preservation of its fine details like edges and textures.
\end{abstract}

Keywords: Ripplet, Directional discrete cosine transform (DDCT), Peak signal to noise ratio, MSE (mean square error), SC (structural content), MD (maximum difference), NCC (normalized cross correlation

Tropical Journal of Pharmaceutical Research is indexed by Science Citation Index (SciSearch), Scopus, International Pharmaceutical Abstract, Chemical Abstracts, Embase, Index Copernicus, EBSCO, African Index Medicus, JournalSeek, Journal Citation Reports/Science Edition, Directory of Open Access Journals (DOAJ), African Journal Online, Bioline International, Open-J-Gate and Pharmacy Abstracts

\section{INTRODUCTION}

Better representation of image details is most important step for executing different image processing tasks like image fusion, image registration [1,2] and image enhancement [3-6]. These image processing tasks serve various fields like medical imaging and pharmaceutical sciences. Medical imaging has emerged as a prominent tool in clinical trials as it enables rapid diagnosis along with visualization and quantitative assessment. The enhancement in the visual quality of medical images like MRI, CT, SPECT, X-Ray image quality lessens the probability of false diagnosis. In medical imaging, image enhancement software is now in great demand. Imaging is a key in medical diagnosis of many diseases and particle size analysis in 
tableting and encapsulation of medicines. The enhancements of images of letters inscribed on medicinal tablets can play a key role in the identification of medicines. In particle size analysis image enhancement technique is used for making particle measurements like particle size, morphology, shape analysis and grayscale or color.

The mapping of source image to coefficients is called a transform. In many image processing tasks, Fourier transform is used to provide an efficient representation of smooth images but not good on images that are dominant with edges. Usually some image features, like edges, cause singularities in the quality of images. Wavelet transform is not capable of resolving discontinuities in two dimensional signals though it is able to resolve the issues regarding one dimensional signal. To overcome the discontinuities, like edges and contours, many transforms such as Ridgelet, Curvelet, Contour let and Ripplet have been devised as wavelet and Fourier transform are unable to resolve edge and contour discontinuities in 2D signal. Ridgelet can resolve 1D discontinuity along vertical and horizontal directions [7]. Ridgelet is based on Radon transform and can provide information of linear edges in image. Although Radon transform is able to extract lines along arbitrary directions, Ridgelet is not capable of resolving singularities in $2 \mathrm{D}$ signals. First generation Curvelet transform was devised by Donoho and it is based on multiscale Ridgelet [7]. Later Donoho proposed second generation Curvelet transform which was able to resolve 2D discontinuities along curves [7]. It uses parabolic scaling law to achieve directionality. Similar approaches like Contourlet and Bandlet were devised to resolve $2 \mathrm{D}$ discontinuities. These new devised image transforms have limitations [7]. While Ridgelet resolves discontinuities only along lines, Curvelet, Contourlet and Ripplet addresses discontinuities along curves. Ridgelet, Curvelet, Bandlet, Brushlet, Contourlet and Ripplet are considered as multiscale geometric analysis (MGA) tools to determine high dimensional signals. These MGA tools have capability to overcome the problems imposed by WT (Wavelet transform). Ripplet is devised using generalization of parabolic scaling law in Curvelet transform. It has six main properties that make it more suitable than Wavelet and its variants in image transformation. These properties are multiresolution, good-localization, high directionality, scaling and support in arbitrary degrees, anisotropy and fast decay of coefficients [5]. Ripplet is capable of providing an efficient representation of image along smooth curves and provides better representation of edges in images. To overcome the limitation of conventional discrete cosine transform, directional discrete cosine transform has been proposed. Conventional discrete cosine transform performs well on horizontal and vertical edges which causes some defects in other directions like diagonal directions whereas Directional Discrete Cosine transform operates on edges in different directions within a specified block size [13]. To evaluate the efficiency of Ripplet and Discrete Cosine transform, experiments on magnetic resonance imaging (MRI) image were conducted. It is quite evident from the literature that Ripplet can represent images more efficiently than Discrete Wavelet transform and Discrete Cosine transform [7]. In this paper we proposed a novel technique of combining RT and DDCT by direct combination and using multiplication and addition operator for better representation of MRI images.

The objective of this study has been is to explore, implement and compare the performance of Ripplet and conventional/directional discrete cosine transform and their combinational approaches for effectual representation of MRI image. It has been assumed that this novel combination is able to improvise the visual diagnostic quality of MRI images.

\section{METHODS}

\section{Experiment 1}

An MRI image used in medical diagnosis was taken as the input source image. This image was reconstructed using Ripplet type-1 transform so as to enhance as well as preserve the edge information along smooth curves. The source image obtained in spatial domain is first transformed in frequency domain. Then the image function was convolved with Ripplet functions. And finally the resultant image was obtained by inverse transforming the frequency domain function back to spatial domain. Ripplet transform provides anisotropic high directional properties to the image features. The step by step algorithm implementation was done using MATLAB.

\section{Experiment 2}

A copy of source image in experiment 1 was reconstructed by conventional/directional DCT at different modes. This transform operated on edges in different modes namely mode 
$0,1,3,4,5,6,7,8$. Here the modes aids to represent various directional features of the image along with its edges. This necessities the use of directional DCT over conventional DCT. The source image was read and reconstructed using numerous code lines in MATLAB software.

\section{Experiment 3}

Ripplet transform was able to represent the shape of the object but not textures [7]. Both DCT and Ripplet Transform work well on the edges and are able to preserve directional features of an image $[7,11,12]$. Now it was thought that combination of these two transforms would be able to cater the advantages provided by both the transforms in a single algorithm that is to visually and quantitatively enhance the texture details of medical and pharmaceutical images. The input image was therefore first passed through Ripplet at 50000 coefficient value then passed through DCT transform.

\section{Experiment 4}

In this experiment, the image reconstructed with Ripplet type-1 transform at 50000 coefficient value was combined with the same input source image reconstructed by DDCT at all the modes via addition operator which leads to the fusing of the image reconstructed at 50000 coefficient value with results from all the modes of DDCT.

\section{Experiment 5}

Using the similar methodology as used in experiment 4 , the images were combined using multiplicative operator. The other mathematical operators like subtraction and division are not taken into consideration as they don't act as the combinational operators.

\section{Experiment 6}

This was performed in a similar manner as experiment 3 except that first the input image was first passed through DCT then through Ripplet type-1 transform. The sequencing of these two transforms was of significant importance as the second experiment performs better which we will see later in detail.

\section{Performance measurement metrics}

The objective evaluation of the proposed method was done by calculating PSNR, MSE, average difference, maximum difference, normalized cross correlation, structural content, normalized absolute error.
(1) PSNR (Peak signal to noise ratio) $[10,14]$ measured peak error. It was used in quantitative measurement of image enhancement, image restoration, image reconstruction excluding image sharpening and calculated between original and reconstructed image. The higher the PSNR, the better is the compressed or reconstructed image. In [14], it is clearly depicted that at low variances or low noise values the PSNR is higher, as noise keeps on increasing the PSNR value keeps on decreasing. The similar observation is reported in our results that higher the PSNR higher is the image enhancement quality shown in experiment 1 to 6 .

(2) MSE (mean square error) [9,14] represents commutative squared error between compressed and original image. MSE should be low, so that image quality is more close to the original image.

(3) AD (average difference) [14] shows average difference between the pixel value of two images. Ideally it should be 0 .

(4) MD (maximum difference) [14] shows maximum difference between the pixel value of the two images.

(5) NCC (normalized cross correlation) [14]-in image processing application is in which brightness of image can vary due to lighting and exposure conditions, though the image can first normalized.

(6) If SC (Structural content) [14] is low, then image quality is good. It can be seen from findings in [14] that at low variances or low noise values the PSNR has the highest value corresponding to lowest SC value. So it can be said that a high value of PSNR corresponds to a low SC value and high PSNR values implies better image quality. Majorly it can be witnessed from literature that in image quality parametric assessment PSNR and MSE plays a significant role whereas SC and NAE have little significance in image enhancement applications.[14] Structural content is one of the correlation based measurement. It measures closeness or relationship between two digital images which can also be quantified in terms of correlations functions. It measures similarity between two images.

(7) Normalized absolute error (NAE) [14] should be low to achieve better image quality results. The mathematical equations for these objective parameters are depicted in earlier report [14]. It can be seen from Table 1 that when the coefficient value is changed from 10000 to 50000 , the value of PSNR increased and the 
normalized absolute error decreases. Unlike previous reports, various other parameters like $A D, M D, S C, N A E, N C C$ besides PSNR and MSE are used in our study so as to present an overall correlation amongst all the parameters, understand their behavior in various combinations as well as identify the various implication(s) associated with each parameter.

\section{RESULTS}

A carefully chosen image (Figure 1) from medical data was selected from Google database. Figure 1(a) shows original input image i.e. the MRI image with saggital view depicting the soft tissues of brain and figure 1(b-f) shows reconstructed images using Ripplet Type-1 transform while increasing the number of coefficients from 10000 to 50000 . As we increased the coefficient value beyond 50000, the execution time was a lot higher comparatively. Therefore results were not depicted beyond 50000 . The best reconstructed image was formed at 50000 coefficient value (Table 1). Figure 2 shows reconstructed image by conventional and directional DCT at various modes $(0,1,3,4,5,6,7,8)$. The objective evaluation values computed for DCT mode 0 are similar to all the other modes (Table 2). Combination of Ripplet and DCT was done by direct combining method (Ripplet first and DCT afterwards; DCT first and Ripplet afterwards), addition operator, and multiplicative operator. The reconstructed images by direct combining of Ripplet transform first and DCT transform in the cascade manner is provided in Figure 3. Resultant image at 50000 coefficient using Ripplet transform showed the best results (Table 1) and hence only this image was combined with DCT transforms at all modes. In Figure 4 the output of combination of Ripplet and DCT transform by addition operator is shown. Here instead of directly combining both the transforms and applying it on images, the first image transformed using Ripplet at 50000 coefficient value (Figure 1-f) and others by DCT transform at all eight modes (Figure 2(a-h) were added together. Hence Figure 4 shows compounded images. The output of combination of Ripplet and DCT transform by multiplication operator is provided in Figure 5 . In this, the two transformed images by both DCT and other Ripplet transforms are combined. Figure 6 shows the resultant image using DCT first and then Ripplet; that is, the image frequency functions are first convolved with DDCT and then with Ripplet transform. This particular combination yielded the best results as it can be seen from the calculated objective evaluation parameters provided in Tables (1-6).

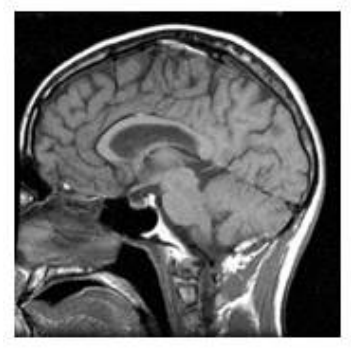

(a)

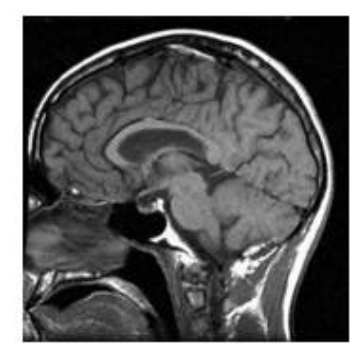

(d)

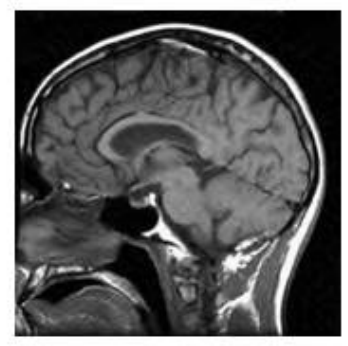

(b)

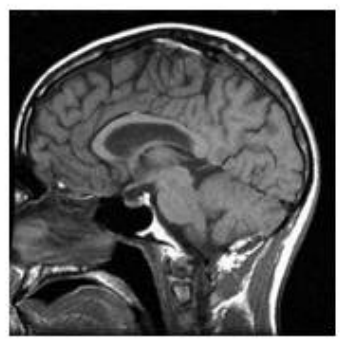

(e)

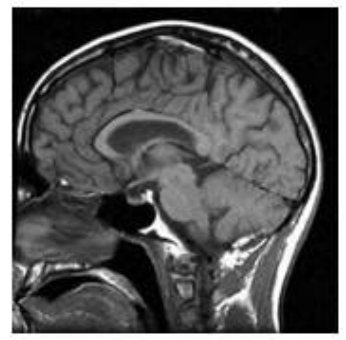

(c)

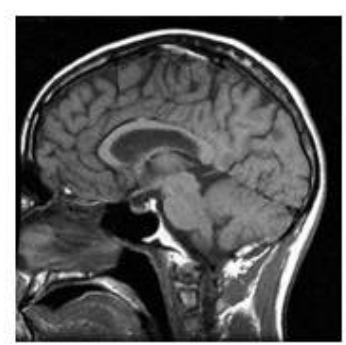

(f)

Figure 1: Original MRI image with saggital view depicting the soft tissues of the brain (a), and reconstructed images by Ripplet transform at 10000 (b), 20000 (c), 30000 (d), 40000 (e), and 50000 (f) respectively

Table 1: Parametric values computed between original and reconstructed images by Ripplet transform at different coefficients 


\begin{tabular}{|c|c|c|c|c|c|c|c|}
\hline $\begin{array}{l}\text { Between } \\
\text { original and } \\
\text { reconstructed } \\
\text { images by } \\
\text { Ripplet } \\
\text { transform at }\end{array}$ & MSE & PSNR & NCC & AD & SC & MD & NAE \\
\hline 10000 coeff. & 75.3459 & 29.3602 & 0.9885 & -0.0372 & 1.0143 & 50 & 0.0943 \\
\hline 20000 coeff. & 50.9499 & 31.0594 & 0.9927 & -0.0218 & 1.0086 & 38 & 0.0785 \\
\hline 30000 coeff. & 43.2900 & 31.7669 & 0.9941 & -0.0190 & 1.0066 & 30 & 0.0724 \\
\hline 40000 coeff. & 40.0985 & 32.0995 & 0.9948 & -0.0347 & 1.0057 & 29 & 0.0697 \\
\hline 50000 coeff. & 38.4702 & 32.2796 & 0.9953 & -0.0424 & 1.0049 & 29 & 0.0681 \\
\hline
\end{tabular}
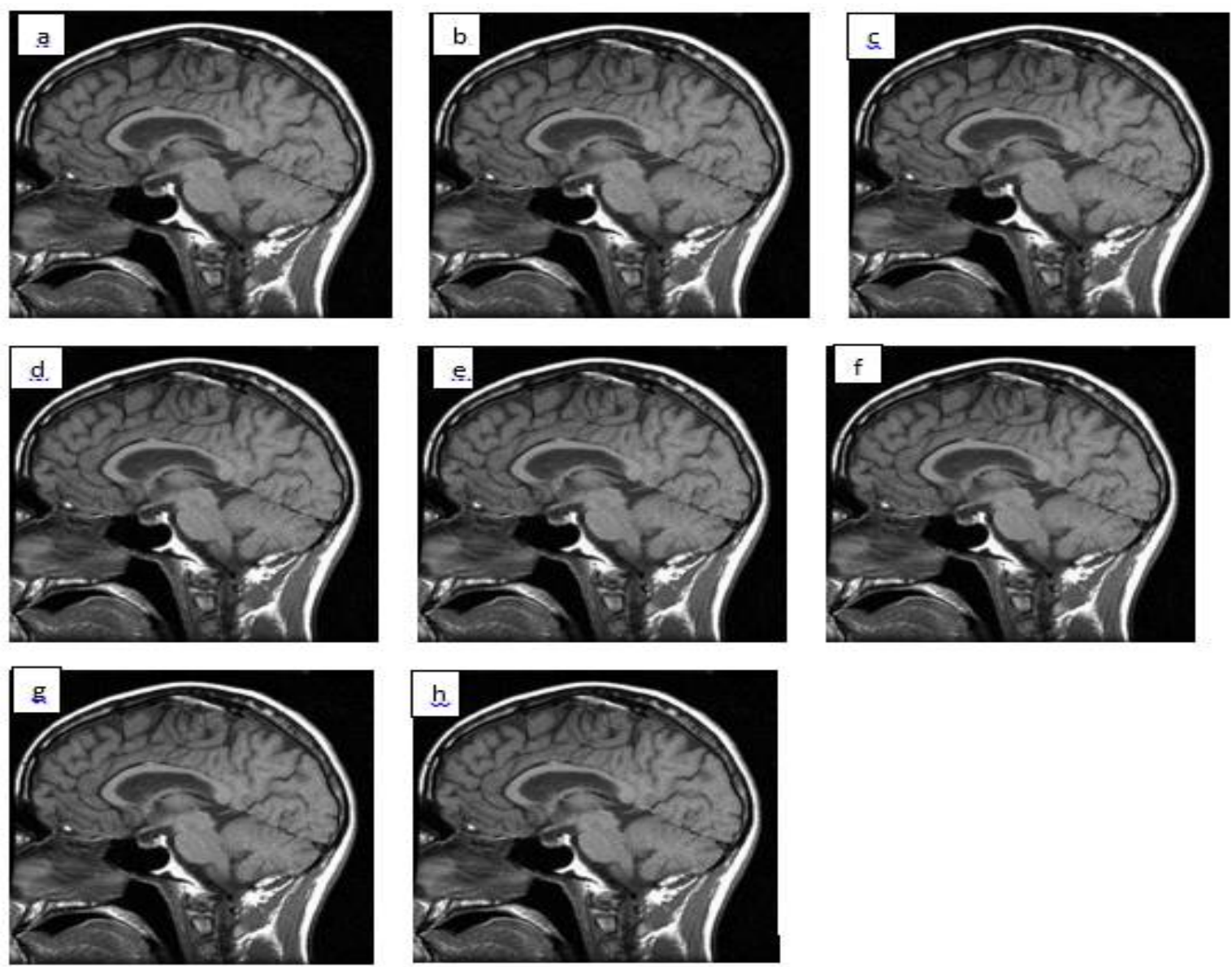

Figure 2: Reconstructed images by conventional and directional DCT at MODE 0 (a), 1 (b), 3 (c), 4 (d), 5 (e), 6 (f), 7 (g) and 8 (h)

Table 2: Parametric values computed between original and reconstructed images by conventional/DDCT transform at different modes

\begin{tabular}{|c|c|c|c|c|c|c|c|}
\hline $\begin{array}{l}\text { Between } \\
\text { original and } \\
\text { reconstructed } \\
\text { images by } \\
\text { DDCT } \\
\text { transform at }\end{array}$ & MSE & PSNR & NCC & AD & SC & MD & NAE \\
\hline mode 0 & 36.9814 & 32.4510 & .9957 & -.0255 & 1.0042 & 34 & .0662 \\
\hline mode1 & 36.9814 & 32.4510 & .9957 & -.0255 & 1.0042 & 34 & .0662 \\
\hline mode 3 & 36.9814 & 32.4510 & .9957 & -.0255 & 1.0042 & 34 & .0662 \\
\hline mode 4 & 36.9814 & 32.4510 & .9957 & -.0255 & 1.0042 & 34 & .0662 \\
\hline mode 5 & 36.9814 & 32.4510 & .9957 & -.0255 & 1.0042 & 34 & .0662 \\
\hline mode 6 & 36.9814 & 32.4510 & .9957 & -.0255 & 1.0042 & 34 & .0662 \\
\hline mode 7 & 36.9814 & 32.4510 & .9957 & -.0255 & 1.0042 & 34 & .0662 \\
\hline mode 8 & 36.9814 & 32.4510 & .9957 & -.0255 & 1.0042 & 34 & .0662 \\
\hline
\end{tabular}



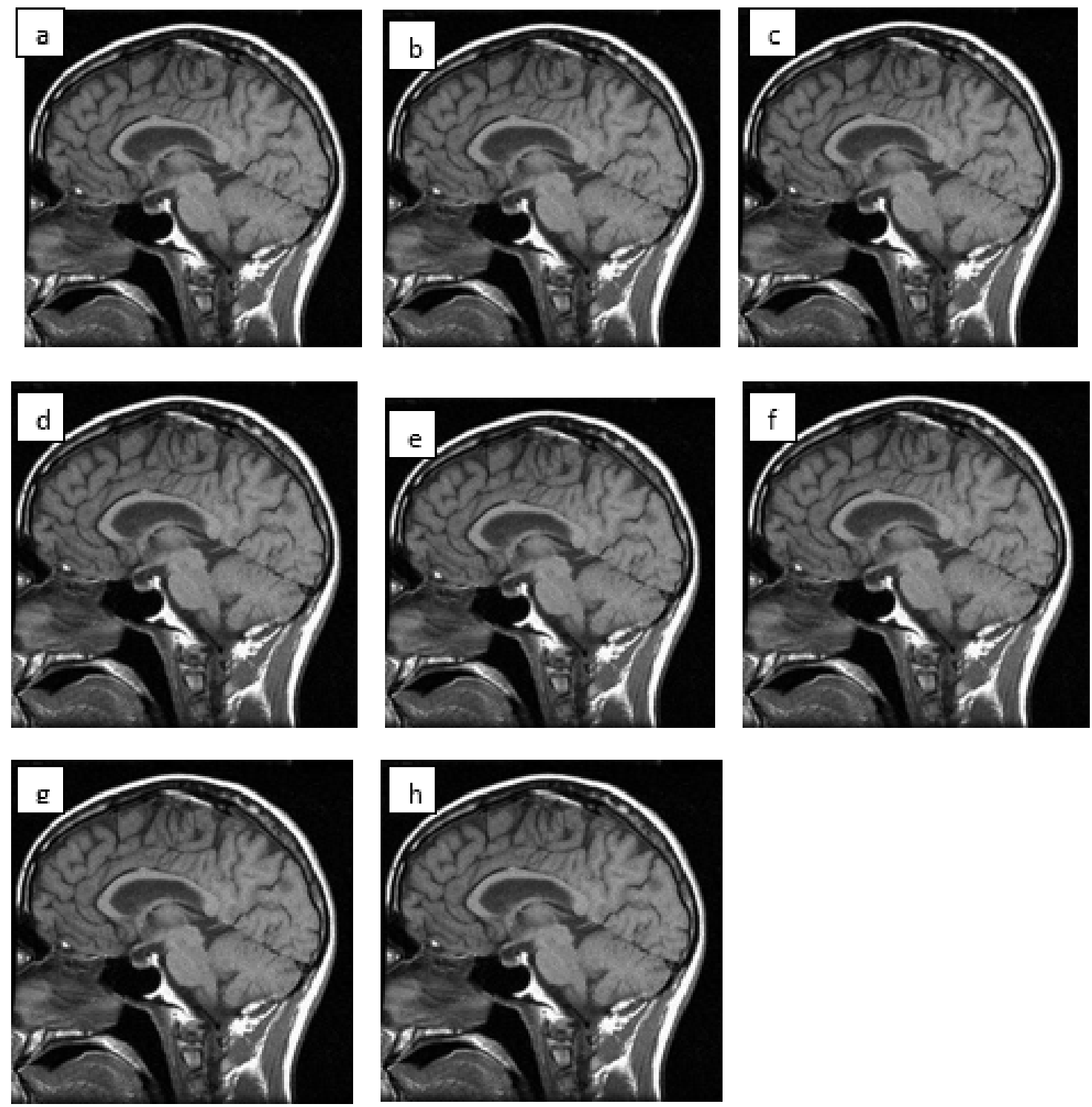

Figure 3: Reconstructed images by combination of Ripplet images at 50000 and DCT at MODE 0 (a), 1 (b), 3 (c), 4 (d), 5 (e), 6 (f), 7 (g) and 8 (h)

Table 3: Parametric values computed between original and reconstructed images by direct combining Ripplet 50000 coefficients and DDCT at different modes

\begin{tabular}{|c|c|c|c|c|c|c|c|}
\hline $\begin{array}{l}\text { Between } \\
\text { original and } \\
\text { reconstructed } \\
\text { images by } \\
\text { direct } \\
\text { combining of } \\
\text { Ripplet and } \\
\text { DDCT at }\end{array}$ & MSE & PSNR & NCC & AD & SC & MD & NAE \\
\hline mode 0 & 38.9035 & 32.2309 & .9946 & -.0388 & 1.0063 & 29 & .0685 \\
\hline mode1 & 38.9035 & 32.2309 & .9946 & -.0388 & 1.0063 & 29 & .0685 \\
\hline mode 3 & 38.9035 & 32.2309 & .9946 & -.0388 & 1.0063 & 29 & .0685 \\
\hline mode 4 & 38.9035 & 32.2309 & .9946 & -.0388 & 1.0063 & 29 & .0685 \\
\hline mode 5 & 38.9035 & 32.2309 & .9946 & -.0388 & 1.0063 & 29 & .0685 \\
\hline mode 6 & 38.9035 & 32.2309 & .9946 & -.0388 & 1.0063 & 29 & .0685 \\
\hline mode 7 & 38.9035 & 32.2309 & .9946 & -.0388 & 1.0063 & 29 & .0685 \\
\hline mode 8 & 38.9035 & 32.2309 & .9946 & -.0388 & 1.0063 & 29 & .0685 \\
\hline
\end{tabular}



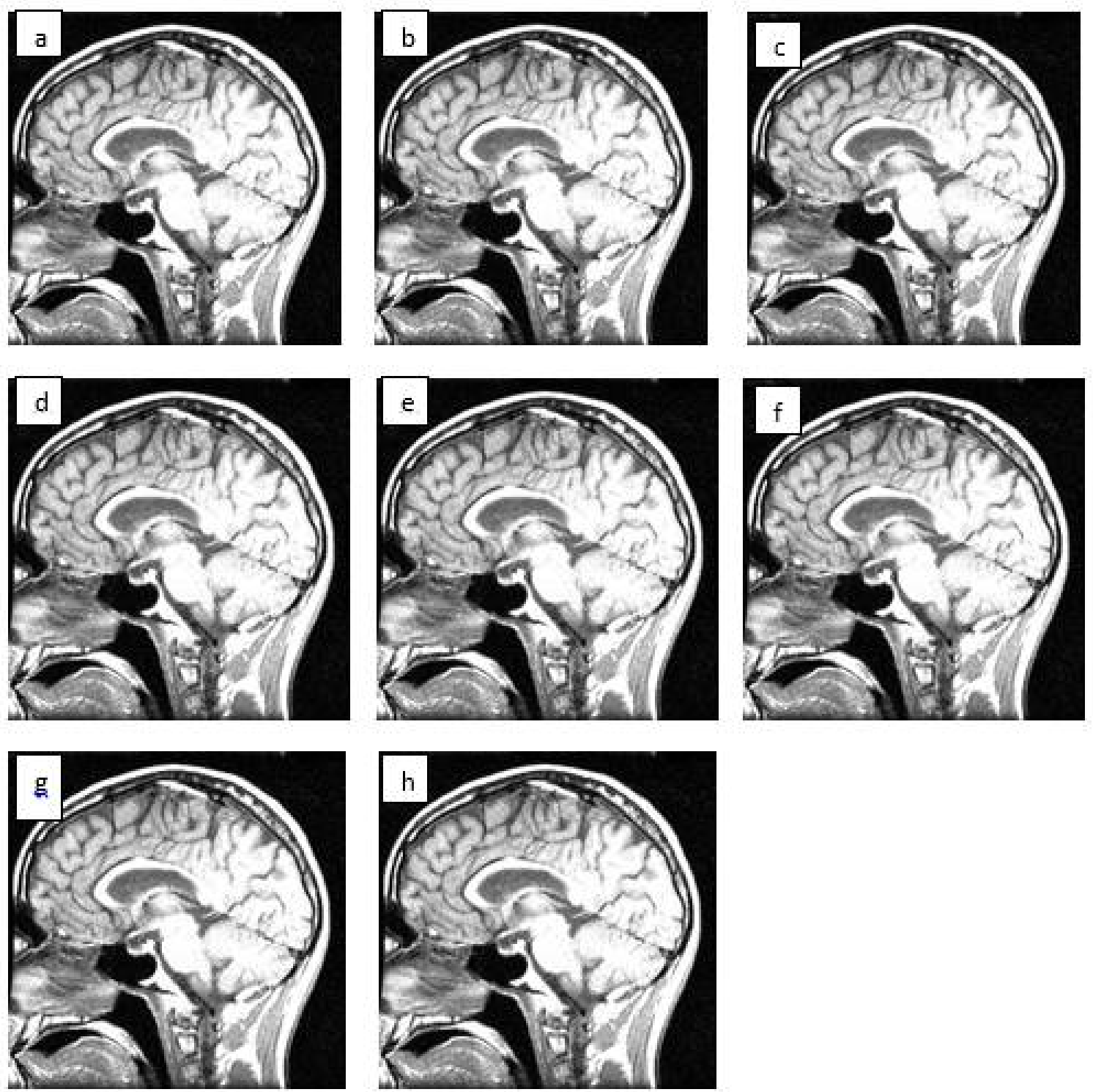

Figure 4: Reconstructed images (a-h) by combination of Ripplet and directional DCT using addition at different modes

Table 4: Parametric values computed between original and reconstructed images by combining Ripplet 50000 coefficients and DDCT at different modes by addition operator

\begin{tabular}{|c|c|c|c|c|c|c|c|}
\hline $\begin{array}{l}\text { Between } \\
\text { original and } \\
\text { reconstructed } \\
\text { images by } \\
\text { Ripplet and } \\
\text { DDCT } \\
\text { combination } \\
\text { by addition } \\
\text { operator }\end{array}$ & MSE & PSNR & NCC & AD & SC & MD & NAE \\
\hline mode 0 & $5.2331 e+03$ & 10.9432 & 1.6623 & -57.6811 & .3400 & 34 & .8242 \\
\hline mode1 & $5.2331 e+03$ & 10.9432 & 1.6623 & -57.6811 & .3400 & 34 & .8242 \\
\hline mode 3 & $5.2331 e+03$ & 10.9432 & 1.6623 & -57.6811 & .3400 & 34 & .8242 \\
\hline mode 4 & $5.2331 e+03$ & 10.9432 & 1.6623 & -57.6811 & .3400 & 34 & .8242 \\
\hline mode 5 & $5.2331 e+03$ & 10.9432 & 1.6623 & -57.6811 & .3400 & 34 & .8242 \\
\hline mode 6 & $5.2331 e+03$ & 10.9432 & 1.6623 & -57.6811 & .3400 & 34 & .8242 \\
\hline mode 7 & $5.2331 e+03$ & 10.9432 & 1.6623 & -57.6811 & .3400 & 34 & .8242 \\
\hline mode 8 & $5.2331 e+03$ & 10.9432 & 1.6623 & -57.6811 & .3400 & 34 & .8242 \\
\hline
\end{tabular}



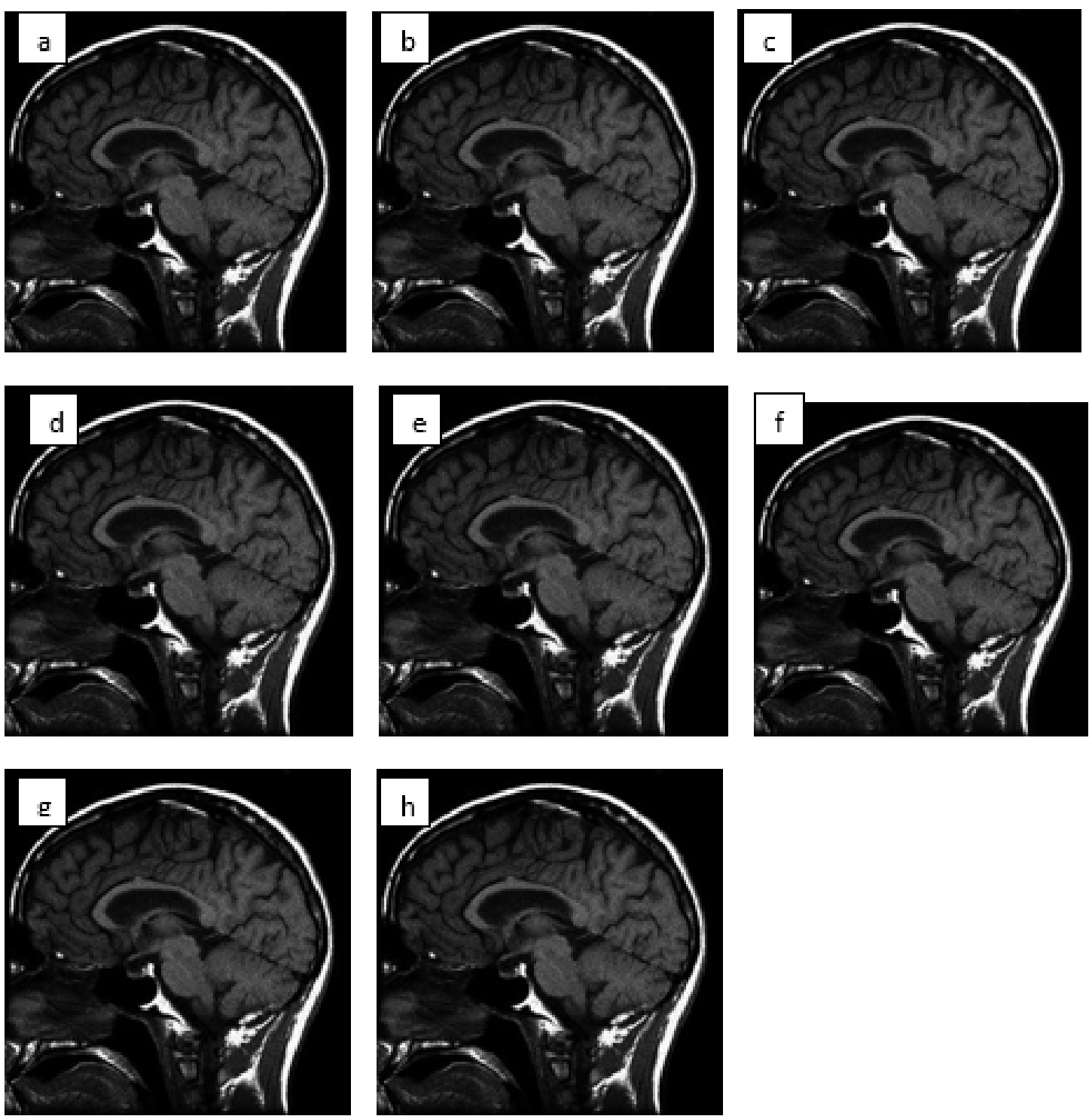

Figure 5: Reconstructed images (a-h) by combination of Ripplet and DDCT using multiplication at different modes $(0,1,3,4,5,6,7,8)$

Table 5: Parametric values computed between original and reconstructed images by combining Ripplet 50000 coefficient and DDCT at different modes by multiplication operator

\begin{tabular}{|c|c|c|c|c|c|c|c|}
\hline $\begin{array}{l}\text { Between } \\
\text { original and } \\
\text { reconstructed } \\
\text { images by } \\
\text { Ripplet and } \\
\text { DDCT } \\
\text { combination } \\
\text { by } \\
\text { multiplcation } \\
\text { operator }\end{array}$ & MSE & PSNR & NCC & AD & SC & MD & NAE \\
\hline mode 0 & $2.0208 e+03$ & 15.0755 & .5803 & 37.2998 & 2.5074 & 92 & .5307 \\
\hline mode 1 & $2.0208 e+03$ & 15.0755 & .5803 & 37.2998 & 2.5074 & 92 & .5307 \\
\hline mode 3 & $2.0208 e+03$ & 15.0755 & .5803 & 37.2998 & 2.5074 & 92 & .5307 \\
\hline mode 4 & $2.0208 e+03$ & 15.0755 & .5803 & 37.2998 & 2.5074 & 92 & .5307 \\
\hline mode 5 & $2.0208 e+03$ & 15.0755 & .5803 & 37.2998 & 2.5074 & 92 & .5307 \\
\hline mode 6 & $2.0208 e+03$ & 15.0755 & .5803 & 37.2998 & 2.5074 & 92 & .5307 \\
\hline mode 7 & $2.0208 e+03$ & 15.0755 & .5803 & 37.2998 & 2.5074 & 92 & .5307 \\
\hline mode 8 & $2.0208 e+03$ & 15.0755 & .5803 & 37.2998 & 2.5074 & 92 & .5307 \\
\hline
\end{tabular}



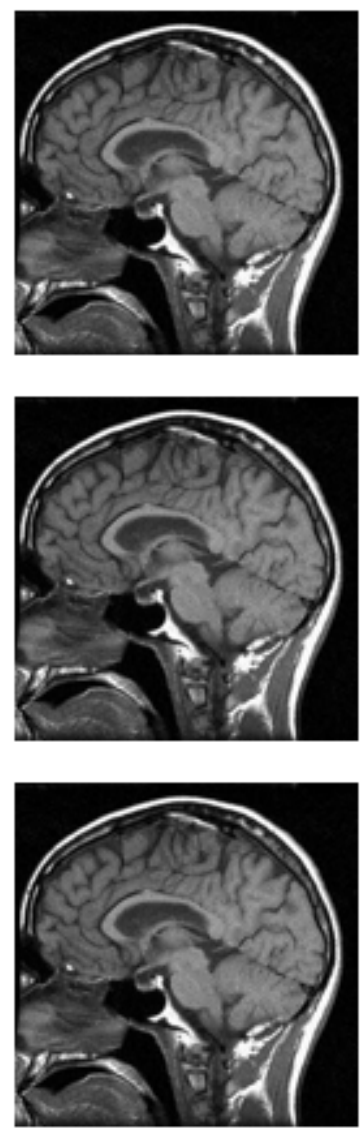
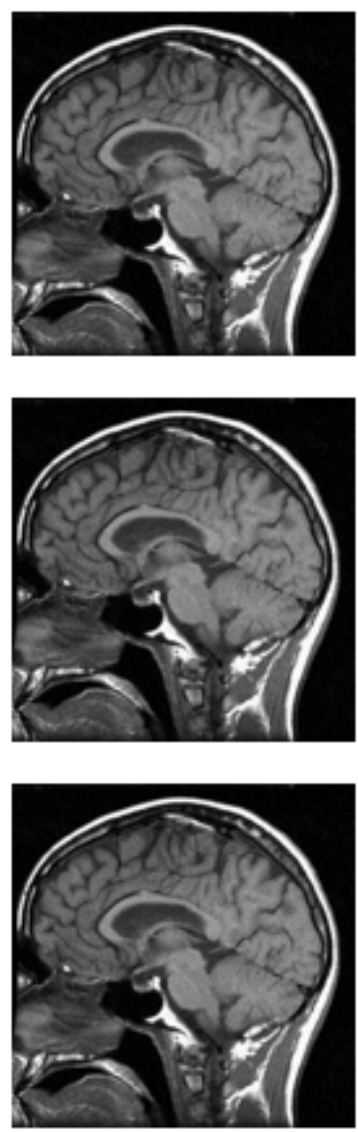
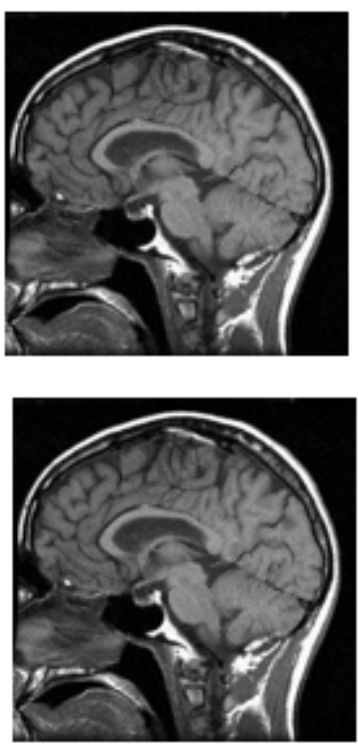

Figure 6: Reconstructed images by combination of DDCT at different modes and Ripplet transform at 50000 coefficient value

Table 6: Parametric values computed between original and reconstructed images by direct combining DDCT at different modes and Ripplet reconstructed images at 50000 coefficient value

\begin{tabular}{|c|c|c|c|c|c|c|c|}
\hline $\begin{array}{l}\text { Between original and } \\
\text { reconstructed images } \\
\text { by direct combining of } \\
\text { DDCTandRipplet } 50000 \\
\text { coefficients at }\end{array}$ & MSE & PSNR & NCC & AD & SC & MD & NAE \\
\hline mode 0 & 37.8405 & 32.3512 & .9948 & -.0172 & 1.0060 & 38 & .0672 \\
\hline mode 1 & 37.8405 & 32.3512 & .9948 & -.0172 & 1.0060 & 38 & .0672 \\
\hline mode 3 & 37.8405 & 32.3512 & .9948 & -.0172 & 1.0060 & 38 & .0672 \\
\hline mode 4 & 37.8405 & 32.3512 & .9948 & -.0172 & 1.0060 & 38 & .0672 \\
\hline mode 5 & 37.8405 & 32.3512 & .9948 & -.0172 & 1.0060 & 38 & .0672 \\
\hline mode 6 & 37.8405 & 32.3512 & .9948 & -.0172 & 1.0060 & 38 & .0672 \\
\hline mode 7 & 37.8405 & 32.3512 & .9948 & -.0172 & 1.0060 & 38 & .0672 \\
\hline mode 8 & 37.8405 & 32.3512 & .9948 & -.0172 & 1.0060 & 38 & .0672 \\
\hline
\end{tabular}

\section{DISCUSSION}

This study has revealed that when an original image was reconstructed using Ripplet type-1 transformation, the image quality increases as the Ripplet coefficient, up to a maximum of 50000 , increases. Although direct DCT produces better image than Ripplet type-1 transformation, some kind of artifacts which can be comprehended by the visual inspection of a radiologist are eminent. By applying DCT first and then Ripplet type-1 transformation, better images are produced.

From the calculated objective evaluation measures (Tables 2-6), it can be seen that the image quality obtained via addition method is inferior to the images obtained by direct combination of transforms but visual inspection is required by radiology expert to determine whether this kind of image can serve significant importance in any kind of medical research or diagnosis (Figure 4). Quantitatively both experiment 4 and 5 i.e. addition and 
multiplication methods are inferior as compared to experiment 3 and 6 (direct combination methods). We can say that addition and multiplication methods are not suitable combinations for better representation of image details. Sometimes the quantitative analysis overshadows the visual quality of images as addition method shows high contrast image in spite of weaker quantitative values as compared to multiplication method.

The reconstructed image in Experiment 1 [Figure 1(b-f) and Table 1] looked closely like the original one. But at the same time, the time taken for coefficient computation and reconstruction increases exponentially with increment in the coefficient value. Memory requirement for storage and bandwidth requirement for transmission would also increase sharply.

From Experiment-2 (Figure-2 and Table-2), we can conclude that DDCT though being a primitive technique has performed better than Ripplet Type-1 transform. From the results given in Table 1 and Table 2 we can clearly deduce that DDCT outperforms Ripplet in terms of PSNR increment and MSE decrement. Also DDCT is able to perform equally well in all eight modes.

The combinational approach of fusing Ripplet and DDCT (Experiment 3, Figure 3, Table 3) is able to produce results which are comparable to original parent techniques along with high degree of feature preservation.

The PSNR of the addition combination is lowest as compared to the results when these transforms were applied individually or in cascade manner. Therefore quantitatively addition method is the most unsuitable combination. According to the objective measurements MD should be as low as possible as it is the pixel difference between the original image and the reconstructed image. Here (experiment-3) it has increased to 34 as compared to 29 in case of direct combination. Also MSE has increased beyond admissible value. All of these facts further support the unsuitability of the addition method. One thing which has to be taken into consideration is the contrast level of the images which has increased drastically which further needs expert radiologist's advice.

From the quantitative results obtained by multiplication method, we can say that the results are better than the addition method and the individual parent techniques. So far we have been able to come to the conclusion that the direct combination of Ripplet and DDCT is the most appropriate method for better representation of medical images. However in experiment- 6 we were able to better results when DDCT is used as the first processing technique and Ripplet as the second processing technique than the former case when Ripplet transform was used as the pre-processing technique. This fact can be looked into more detail from the tabular value of PSNR which has increased when DDCT was applied first i.e. experiment-6. The intuitive idea for using the DDCT as the first transform is that it gives higher PSNR value than the Ripplet transform along with its object depiction capability. Therefore using DDCT as the first case kind of serves an enhanced input image to Ripplet transform to further represent the image details in totality depict the shape of the object as well as the texture details. Thus, the use of DDCT first and followed by Ripplet as the second transform is the best suitable method to depict the shape of the object as well as the texture details.

This study signifies that various transformations can be done on magnetic resonance images to provide an enhanced image which leads to an improvement in the diagnostic quality, while preserving edge features and texture details. The enhanced image holds a lot of importance in medical imaging for accurate and precise diagnosis. This paper thus provide a method enhance MRI images which can be useful in abnormality detection in brain, lesion detection, skull based surgery and ear-nose-and-throat surgical procedures. However, further studies are needed to modify the selection of transforms for various combinations and many features could be targeted besides edges and textures. The future analysis could be done on CT images, PET images, SPECT images X-RAY and ultrasound imagery. Also the possibility of occurrence of various kinds of artifacts can be taken into consideration in further studies.

\section{CONCLUSION}

The reconstruction of images appears to be better with DCT rather than Ripplet transform though DCT is a more primitive technique than Ripplet transform. After this, the expedition of combining of both of these techniques was done. These individual image transformation techniques often do not often produce very good image texture details. Direct combination of RT and DDCT transforms in sequence provide much better images.

Although Ripplets are capable of representation of the shape of an object, but they are not apt as 
it comes to the representation of textures. Though the experiments have been done by combining the Ripplet transform with DCT, much better results may be obtained by trying various combinations of Ripplet with other transforms like direction -lets, Ridgelet, Curvelet and Contourlet.

\section{DECLARATIONS}

\section{Conflict of Interest}

No conflict of interest associated with this work.

\section{Contribution of Authors}

The authors declare that this work was done by the authors named in this article and all liabilities pertaining to claims relating to the content of this article will be borne by them.

\section{REFERENCES}

1. Dogra A and MS Patterh. "CT and MRI Brain Images Registration for Clinical Applications." J Cancer Sci Ther 6 (2014): 018-026.

2. Dogra $A$ and $P$ Bhalla. "CT and MRI Brain Images Matching Using Ridgeness Correlation."

3. Dogra A and P Bhalla. "Image Sharpening By Gaussian and Butterworth High Pass Filter."

4. Dogra A and A Dogra. "Performance Comparison of Gaussian and Elliptic High Pass Filter." International journal of Advanced Biological and Biomedical Research 3.1 (2015): 93-96.
5. Dogra, A., and Sunil Agrawal. "Efficient Image Representation Based on Ripplet Transform and PureLet." Int. J. Pharm. Sci. Rev. Res., 2015: 34(2): 93-97.

6. Dogra, A., and Sunil Agrawal. "3-Stage Enhancement Of Medical Images Using Ripplet Transform, High Pass Filters and Histogram Equalization Techniques". International Journal Of Pharmacy And Technology, Dec 2015 Vol. 7 Issue No.3 9748-9763.

7. Xu, Jun, Lei Yang, and Dapeng Wu. "Ripplet: A new transform for image processing." Journal of Visual Communication Representation 21.7 (2010): 627-639.

8. Das, Sudeb, Manish Chowdhury, and Malay Kumar Kundu. "Medical image fusion based on ripplet Transform type-I." Progress In Electromagnetics Research B 30 (2011): 355-370

9. Wang, Zhou, and Alan C. Bovik. "Mean squared error: love it or leave it? a new look at signal fidelity Measures." Signal Processing Magazine, IEEE 26.1 (2009): 98-117

10. Mathworks.in/help/vision/ref/psnr.html

11. Rao K. R. and Yip P.. Discrete Cosine Transform Algorithms, Advantages, Applications. London: Academic Press, 1990

12. Ahmed N., Natarajan T., and Rao K. R.. "Discrete cosine transform," IEEE Trans. Computer, vol. 23, pp. 90-93, 1974.

13. Kauff P. and Schuur K.. "Shape-adaptive DCT with block based DC separation and $\square D C$ correction". IEEE Trans. Circuits and Systems for Video Technology, vol. 8, pp. 237-242, June 1998.

14. Ravi $K$ and Munish R. "Analysis of various quality metrics medical image processing". IJARCSSE, Volume 2, issue 11, pp.137-143, November-2012. 